\title{
Consumer views of portion size estimation aids
}

\author{
G. P. Faulkner ${ }^{1}$, L. K. Pourshahidi ${ }^{1}$, M. Dean ${ }^{2}$, M. Spence ${ }^{2}$, S. O’Brien ${ }^{3}$, E. Gibney ${ }^{3}$, M. A. Kerr ${ }^{1}$, \\ T. A. McCaffrey ${ }^{1}$, J. M. W. Wallace ${ }^{1}$ and M. B. E. Livingstone ${ }^{1}$ \\ ${ }^{1}$ Northern Ireland Centre for Food and Health, University of Ulster, Coleraine, BT52 1SA, ${ }^{2}$ School of Biological Sciences, \\ Queen's University Belfast, BT9 5AG and ${ }^{3}$ Institute of Food and Health, University College Dublin, Belfield, Dublin 4, \\ Ireland
}

Larger portion sizes (PS) are one of many factors contributing to the rise in obesity, and this has also distorted consumer's perceptions of appropriate $\mathrm{PS}^{(1)}$. To date, there has been little research into consumers' views about portion size estimation aids (PSEA) available to assist with estimating more appropriate PS. The current study aims to explore consumer preferences for PSEA.

Six focus groups (three in NI and three in ROI) with three to eight participants in each group were formed. In total, 32 participants (17 males; 15 females, aged 18-64 years) from both urban and rural locations and representing a range of socio-economic groups took part. Their views on a representative range of PSEA were explored using a semi-structured discussion guide. PSEA included: quantities and measures (e.g. serving sizes on food labels), reference objects (e.g. palm of the hand), household measures and utensils (e.g. $200 \mathrm{ml}$ disposable cup), and food packaging (e.g. demarcations on cheese block). The focus groups were audio recorded, professionally transcribed verbatim, coded by two researchers independently and analysed using an inductive thematic analysis ${ }^{(2)}$.

The major themes which emerged are outlined below with example quotes:

\begin{tabular}{ll}
\hline Major themes & Example quotes from participants \\
\hline Prefer 'visuals' (objects/utensils/ indicators on & "I think the visual is better than ... you know when you can actually \\
food packaging) to quantities & see the amount rather than grams" \\
Use PSEA before cooking food & "Before I start cooking I would measure the rice" \\
More realistic serving sizes & "It says 18 servings per box; I would say I would get half of that out of a box, cereals especially..." \\
PSEA are only for weight loss & "Yeah, if you start at school and then you grow up yourself with it" \\
Introduce early and reinforce & "Not fruit and veg... all fruit and veg are free in our house, you can have as much as it as you want" \\
PSEA needed for grains not fruit and veg & "But what about a man that's on a diet that's obese against a normal man that's active?" \\
Account for age, gender and activity level & "That would be alright if you knew the number of servings" \\
Additional guidance on no. of servings per day (range) & "Leaflets and posters I don't know whether you pay much attention to them" \\
Via TV ads/ free mobile app not leaflet (routes for communication) & "But from a single source. We've seen lots of contradictory messages..." \\
Consistent serving sizes from one authoritative body &
\end{tabular}

This study has identified consumer preferences for "visual" PSEA (e.g. reference objects, household measures and utensils, and food packaging) communicating realistic serving sizes which are flexible for individual requirements, presented consistently and introduced innovatively at an early age. Stakeholders should be cognisant of such views on PSEA when promoting their use for more effective management of PS selection.

This material is based upon works supported by safefood, the Food Safety Promotion Board, under Grant No. 07-2010. Ethical approval was obtained from the Biomedical Sciences Ethics Filter Committee, University of Ulster and the study was conducted according to the guidelines laid down in the Declaration of Helsinki.

1. Jeffery RW, Rydell S, Dunn CL et al. (2007) Int J Behav Nutr Phys Act, 4: 27.

2. Braun V \& Clarke V (2006) Qual Res Psychol 3, 77-101. 